This isoenzyme was rapidly inactivated at $70^{\circ} \mathrm{C}$ $\left(t_{\frac{1}{2}} \simeq 2.5 \mathrm{~min}\right)$, was inactive with quinate and formed part of the biosynthetic enzyme aggregate of molecular weight 280000 . Extracts from mycelia grown with shikimate or quinate showed in addition much larger amounts of an inducible dehydroquinase. This inducible isoenzyme could be separated from the biosynthetic isoenzyme by sucrose-density-gradient centrifugation $\left(s_{20, w} 9.2 \mathrm{~S}\right)$ and was thermostable at $70^{\circ} \mathrm{C}\left(t_{t}>1 \mathrm{~h}\right)$. These two treatments followed by DEAE-cellulose chromatography and Sephadex G-200 chromatography led to an almost homogeneous preparation of the inducible enzyme.

This isoenzyme had molecular weight 180000 and optimum pH8.0. The $K_{m}$ for 5-dehydroquinate was $0.42 \mathrm{~mm}$; the enzyme was also active against quinate with $K_{m} 46 \mathrm{~mm}$, a feature in which it differed from the biosynthetic isoenzyme.

Tresguerres et al. (1970) showed that protocatechuate was the product inducer for the quinate/ shikimate dehydrogenase in Acinetobacter calcoaceticus. In Asp. niger protocatechuate had no such function, but was, in contrast, a powerful competitive inhibitor of the inducible dehydroquinase $\left(K_{t}\right.$ $0.18 \mathrm{~mm}$ ), providing a likely point for regulatory control. Only shikimate, quinate or compounds containing a shikimate or quinate moiety induced the dehydrogenase and the inducible dehydroquinase isoenzyme. A mutant lacking the inducible dehydroquinase still grew with quinate, presumably by using the biosynthetic thermolabile isoenzyme to produce dehydroshikimate and thence protocatechuate. The latter was degraded via the 3-oxoadipate pathway and was the inducer for the first three enzymes of this route.

This study was performed during the tenure of a Royal Society Japan Fellowship and was further supported in Kyoto by a Sigma Research Award.

Ahmed, S. I. \& Giles, N. H. (1969) J. Bacteriol. 99, 231

Cain, R. B. (1972) Biochem. J. 127, 15P

Giles, N. H., Partridge, C. W. H., Ahmed S. I. \& Case,

M. E. (1967) Proc. Nat. Acad. Sci. U.S. 58, 1930

Gross, S. R. (1958) J. Biol. Chem. 233, 1146

Tresguerres, M. E. F., de Torrontegui, G., Ingledew, W. M. \& Canovas, J. L. (1970) Eur. J. Biochem. 14, 445

\section{Biochemical Investigations on Conidiation of Aspergillus nidulans in Submerged Liquid Culture}

By S. D. MARTINelli (Department of Genetics, University of Cambridge, Cambridge CB4 $1 X D, U . K$.)

Aspergillus nidulans undergoes both vegetative growth and asexual development in a defined minimal medium. Cultures are shaken at $37^{\circ} \mathrm{C}$, at $220 \mathrm{rev} /$ min and with glucose as the limiting nutrient in the standard medium.

$\mathrm{Up}$ to $10^{8}$ conidia $/ \mathrm{ml}$ are produced in these conditions. A burst of conidial production commences $5 \mathrm{~h}$ after germination is complete, during the deceleration phase of growth. Very few conidia are produced during autolysis, but a balance is maintained between these and conidia that germinate. The conidial heads in liquid medium are smaller and appear to produce fewer conidia per head than on solid medium. Vesicles are not apparent, only structures resembling phialides, which are mainly terminally produced and a few in intercalary positions. Conidial chains of more than two are not produced.

The strain used has a $p$-diphenol oxidase whose end product is the green conidial pigment. This enzyme appears in both the mycelium and the medium. The enzyme reaches a maximum specific activity $\mid 36 \mathrm{~h}$ after inoculation. About the same time the conidia become pigmented green. Similar results have been obtained with colonies grown on solid medium (Clutterbuck, 1969).

The enzyme is first detectable in hyphal extracts at the same time as conidial production. There is a delay of $2-3 \mathrm{~h}$ before the enzyme appears in the medium, coinciding with visible signs of autolysis. It is possible that the enzyme is active outside the cell membrane (A. J. Clutterbuck, personal communication). The specific activity of the enzyme in the medium is much higher than that in the hyphal extracts.

By lowering the glucose concentration from 1 to $0.1 \%$ the dry weight, $p$-diphenol oxidase activity and conidial production are all decreased, but disproportionately. The dry weight is decreased by onetenth, the conidial production by one-half and no enzyme activity is detectable. In this medium conidia are produced either on the germination hyphae or on primary branchs.

Varying the concentrations of nitrogen sources $\left(\mathrm{NO}_{3}{ }^{-}\right.$or $\left.\mathrm{NH}_{4}{ }^{+}\right)$concomitantly changes dry weight and conidial production. The ratio of conidia produced per $\mathrm{mg}$ of dry weight is greater for $\mathrm{NH}_{4}{ }^{+}$than for $\mathrm{NO}_{3}-$ than for urea.

Clutterbuck, A. J. (1969) Heredity 24, 515 